Article

\title{
Characterization of Volatile Organic Compounds of Healthy and Huanglongbing-Infected Navel Orange and Pomelo Leaves by HS-GC-IMS
}

\author{
Shan Cao ${ }^{\dagger}$, Jingyu Sun ${ }^{\dagger}$, Xiaoyong Yuan, Weihui Deng, Balian Zhong and Jiong Chun * \\ National Navel Orange Engineering Research Center, College of Life Sciences, Gannan Normal University, \\ Ganzhou 341000, China; scoral29116@163.com (S.C.); SJYnj_1997@163.com (J.S.); \\ gzyuanxiaoyong@163.com (X.Y.); dwh110by@163.com (W.D.); bal.zh@163.com (B.Z.) \\ * Correspondence: qczx99@163.com; Tel.: +86-797-839-3068 \\ + These authors contributed equally to this work. \\ Academic Editors: Laura De Martino and Derek J. McPhee \\ Received: 4 August 2020; Accepted: 4 September 2020; Published: 9 September 2020

\begin{abstract}
The Asian citrus psyllid (ACP), Diaphorina citri Kuwayama, is the only natural vector of bacteria responsible for Huanglongbing (HLB), a worldwide destructive disease of citrus. ACP reproduces and develops only on the young leaves of its rutaceous host plants. Olfactory stimuli emitted by young leaves may play an important role in ACP control and HLB detection. In this study, volatile organic compounds (VOCs) from healthy and HLB-infected young leaves of navel orange and pomelo were analyzed by headspace-gas chromatography-ion mobility spectrometry (HS-GC-IMS). A total of 36 compounds (including dimers or polymers) were identified and quantified from orange and 10 from pomelo leaves. Some compounds showed significant differences in signal intensity between healthy and HLB-infected leaves and may constitute possible indicators for HLB infection. Principal component analysis (PCA) clearly discriminated healthy and HLB-infected leaves in both orange and pomelo. HS-GC-IMS was an effective method to identify VOCs from leaves. This study may help develop new methods for detection of HLB or find new attractants or repellents of ACP for prevention of HLB.
\end{abstract}

Keywords: Huanglongbing; navel orange; pomelo; leaf volatiles; HS-GC-IMS; principal component analysis (PCA)

\section{Introduction}

Huanglongbing (HLB), also known as citrus greening disease, is a worldwide destructive disease of citrus [1]. HLB has caused several billion dollars in losses to the citrus industry in Florida, USA: Citrus-bearing acres have decreased from 679,000 in 2003-04 to 402,000 in 2017-18 and the number of citrus growers went down from 7389 in 2002 to 2775 in 2017 [2]. HLB-infected citrus trees have yielded fewer and poorer-quality fruits, which are less juicy, and bitter and metallic in taste [3,4]. The Asian citrus psyllid (ACP), Diaphorina citri Kuwayama, is the only vector of bacteria responsible for HLB [5]. Novel and sustainable approaches to the control of ACP are urgently needed for successful HLB management programs.

ACP mates, oviposits, and develops exclusively on new flush shoots [6]. Recent studies have shown that volatile organic compounds (VOCs) emitted by flushing shoots may play an important role in the detection, location, and evaluation of potential host plants by ACP [7]. The ability to understand the chemical composition of citrus leaf VOCs may facilitate ACP's ability to recognize the stimuli signal from its host plant and the interaction between them. Wenninger et al. demonstrated that ACP used olfactory cues in orientation to host plants and suggested using plant VOCs to monitor and 
manage ACP [8]. Robbins et al. used GC-MS to identify compounds in headspace volatiles collected from uninfected flush and young leaves of various citrus genotypes [9]. Alquézar et al. found that (E)- $\beta$-caryophyllene, a VOC from guava, exerts a repellent effect on ACP [10]. Andrade et al. studied the chemical composition of volatile oils from 22 genotypes of citrus and related genera and speculated that phytol, (Z)- $\beta$-ocimene, and $\beta$-elemene may act as repellents to ACP [11].

In spite of finding efficient repellents or attractants to control ACP, detection of HLB is also very important. Currently, polymerase chain reaction (PCR) is widely used for HLB detection [12,13]. However, this technique has limitations because of the low concentration of bacteria in the infected trees, and HLB symptoms may not appear on leaves for a long time after infection [14]. An alternative approach for detection of HLB is monitoring the VOCs emitted by the plant, which may serve as an indicator to discriminate healthy and infected trees. Olfactory cues have been shown to be important in HLB detection. Aksenov et al. illustrated that changes in VOCs, including linalool, tetradecane, and phenylacetaldehyde, were correlated with HLB-infected trees at the asymptomatic stage [15]. Wang et al. reported gas biosensor arrays for the detection of VOCs released by HLB-infected citrus trees [16]. The typical techniques for VOC detection utilize gas chromatography (GC) in combination with various detectors, including flame ionization (GC-FID), mass spectrometry (GC-MS), and differential mobility spectrometry (GC-DMS) [17].

China is one of the world's leading producers of citrus [18]. The Newhall navel orange industry in Gannan of China covers an area of 0.29 million acres with a total annual output of more than one million tons of oranges. Pomelo (Citrus maxima (Burm.) Merr.) is also a popular citrus variety cultivated in Southern China with an annual yield around 0.5 million tons [19]. HLB is the number one threat and responsible for major economic loss to citrus production in China. Monitoring plant health and detecting infection are important to reducing HLB spread and facilitating effective management practices. There are many methods and devices that have been reported to extract and analyze VOCs in plant leaves, such as simultaneous distillation-extraction (SDE) [20], steam distillation (SD) [21], and supercritical fluid extraction (SFE) [22]. Wang et al. analyzed the components of volatiles from the new shoots of six healthy host plant species via the headspace solid-phase microextraction (HS-SPME) method and showed that $\beta$-caryophyllene was the characteristic VOC in flushing shoots of the tested ACP host plant species [23]. However, they did not analyze VOCs from HLB-infected species. Sharma et al. used a portable GC device for rapid, in situ, dynamic monitoring of the VOCs produced by milkweeds under aphid attack [24]. New technologies with greater reliability, precision, and accuracy are needed to detect HLB and control ACP. In our study, young leaves of two varieties of citrus, Gannan Newhall navel orange and Shatian pomelo (Citrus maxima (Burm.) Merr. cv. Shatian $\mathrm{Yu}$ ): Healthy orange leaf (HEAO), Huanglongbing-infected orange leaf (HLBO), healthy pomelo leaf (HEAP), and Huanglongbing-infected pomelo leaf (HLBP), were chosen as studying samples. The headspace-gas chromatography ion mobility spectrometry (HS-GC-IMS) technique was used to identify the composition difference between healthy and HLB-infected leaves.

Ion mobility spectrometry (IMS) is a powerful and accurate analytical technique with high sensitivity and simplicity, which hardly requires sample preparation steps [25]. It can detect and characterize chemical substances based on the different migration rates of gas-phase ions under an electric field. The IMS technique has been employed widely and successfully to detect chemicals in agricultural analysis, foods, and pharmaceutics [26]. It has been proved to be a very effective technique for the identification and quantification of VOCs with different properties in the gas phase [27]. Combining IMS with GC is a more efficient method to make better use of its advantages, especially improving the analytical selectivity of complex samples [28]. Headspace-gas chromatography ion mobility spectrometry (HS-GC-IMS) is the technique that combines GC-IMS with headspace sampling. It could be used to characterize VOCs in different samples with low detection limits and good selectivity $[29,30]$.

In this study, we conducted a preliminary investigation of VOCs emitted by the young leaves of navel orange and Shatian pomelo, which are representative ACP host plants. The volatile fingerprints 
of healthy orange leaf (HEAO), pomelo leaf (HEAP), Huanglongbing-infected orange leaf (HLBO), and pomelo leaf (HLBP) samples were established by HS-GC-IMS. The use of HS-GC-IMS to analyze VOCs in orange and pomelo leaf samples and distinguish healthy and HLB-infected leaves has not been reported. This work might provide a reference to develop a new method for detection of HLB and find new attractants or repellents of ACP for prevention of HLB.

\section{Results and Discussion}

\subsection{HS-GC-IMS Topographic Plots of HEAO, HLBO, HEAP, and HLBP}

The information of VOCs of HEAO, HLBO, HEAP, and HLBP were obtained via HS-GC-IMS analysis. A 3D spectrum was generated by a Flavor Spec ${ }^{\circledR}$ instrument, as shown in Figures 1 and 2. The $\mathrm{X}$-axis denotes the ion drift time, the $\mathrm{Y}$-axis denotes the retention time of the gas chromatograph, and the Z-axis denotes the peak intensity in the topographic map. The VOCs in different samples demonstrated varying peak intensities. Number 1, 2, 3 indicate triplicate experiments, for example, HEAO1, HEAO2, and HEAO3 were triplicate experiments of HEAO. HLBO had more peak signals of VOCs than HEAO, and HLBP had more peak signals of VOCs than HEAP. A study on the changes of metabolites in citrus leaves in response to ACP stress might be helpful for HLB detection and ACP control.

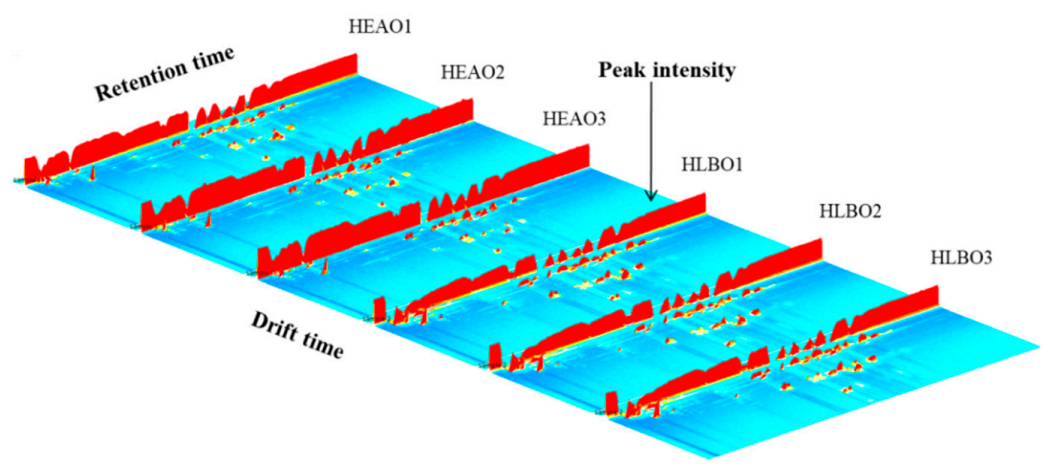

Figure 1. Three-dimensional topographic images of VOCs from healthy and Huanglongbing (HLB)-infected orange leaves (healthy orange leaf (HEAO) and Huanglongbing-infected orange leaf (HLBO)). Number 1, 2, 3 indicate triplicate experiments.

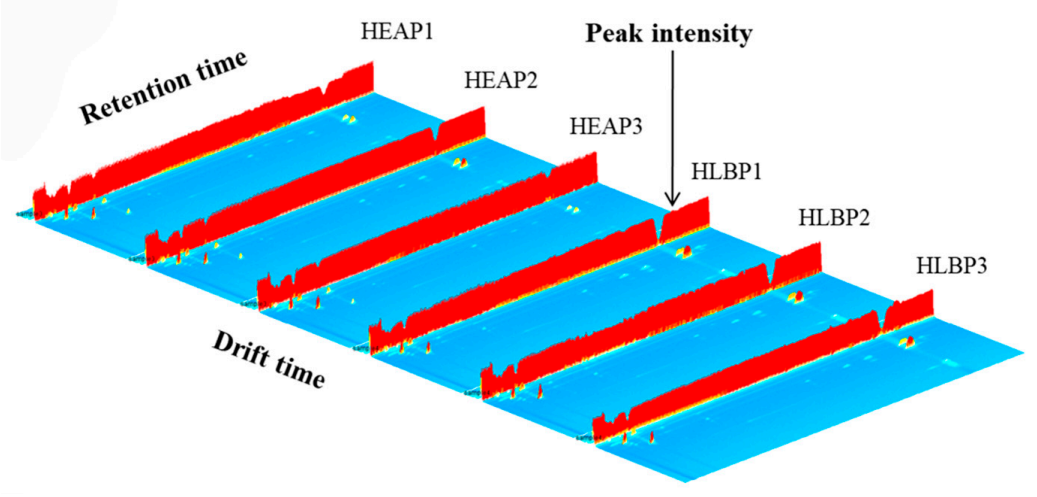

Figure 2. Three-dimensional topographic images of VOCs from healthy and HLB-infected pomelo leaves (healthy pomelo leaf (HEAP) and Huanglongbing-infected pomelo leaf (HLBP)). Number 1, 2, 3 indicate triplicate experiments.

For the convenience of comparison, a vertical view was used as shown in Figure 3. The background of HEAO1 is blue, and the red vertical line at horizontal coordinate 1.0 is the reactant ion peak (RIP, normalized drift time of 7.93). Each point on the right side of RIP represents a VOC. The spectral diagram of HEAO1 was selected as the reference, while the spectral diagram of other samples was 
deducted from the reference. If two VOCs were identical, the background after deduction would be white. Peak intensities are indicated by different colors. Red spots indicate a higher concentration of the VOCs than the reference, whereas blue spots indicate a lower concentration of the VOCs. The data were displayed at the topographic plot zone with a retention time from 100 to $1000 \mathrm{~s}$ and drift time (RIP relative) from 1.0 to 2.5. It is obviously shown that HLBO had more VOC peak signals, and most VOCs had a higher concentration than HEAO.

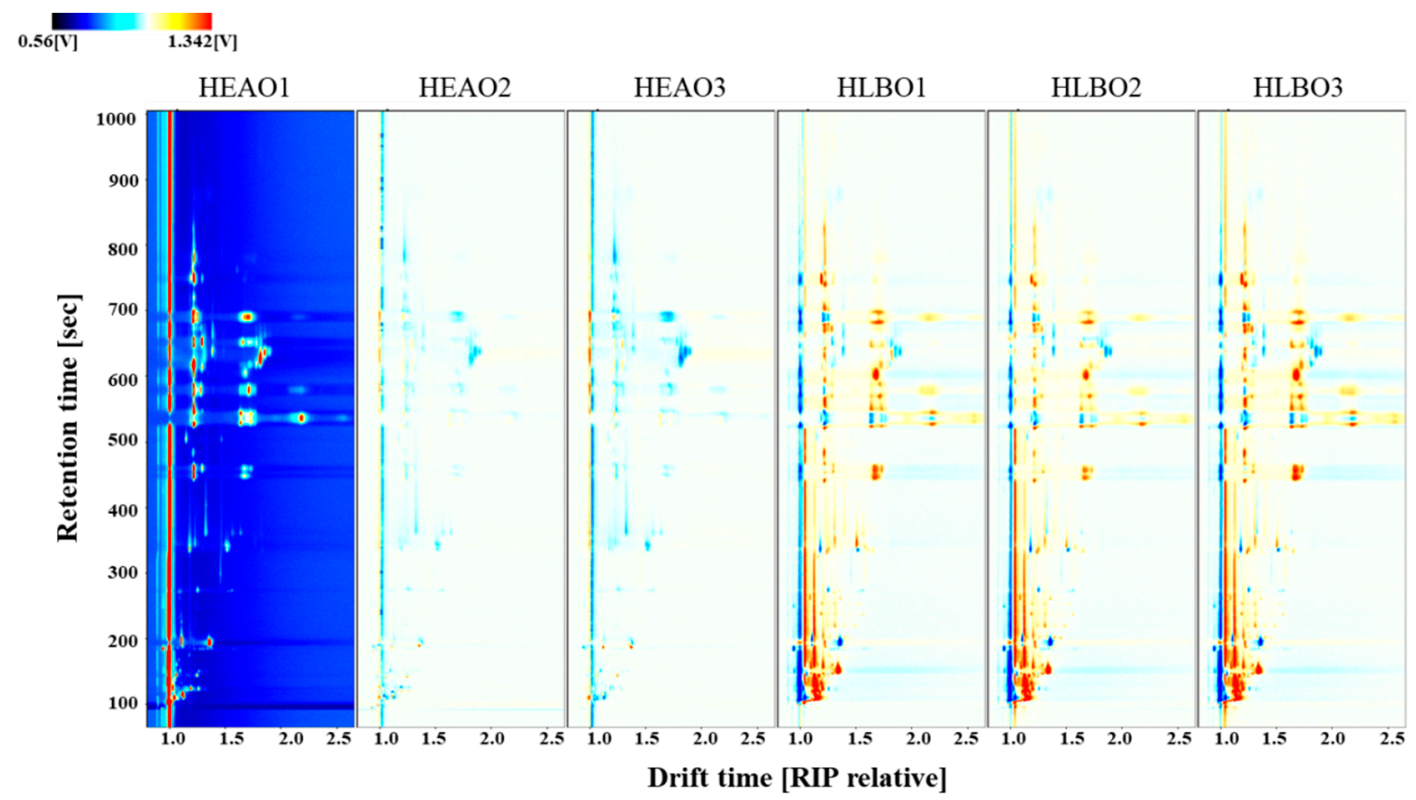

Figure 3. Comparison of ion migration chromatogram of volatile organic compounds from healthy and HLB-infected orange leaves (HEAO and HLBO).

A comparison of volatile organic compounds from HEAP and HLBP is shown in Figure 4. It is obviously shown that HLBP had more VOC peak signals and most VOCs had a higher concentration than HEAP.

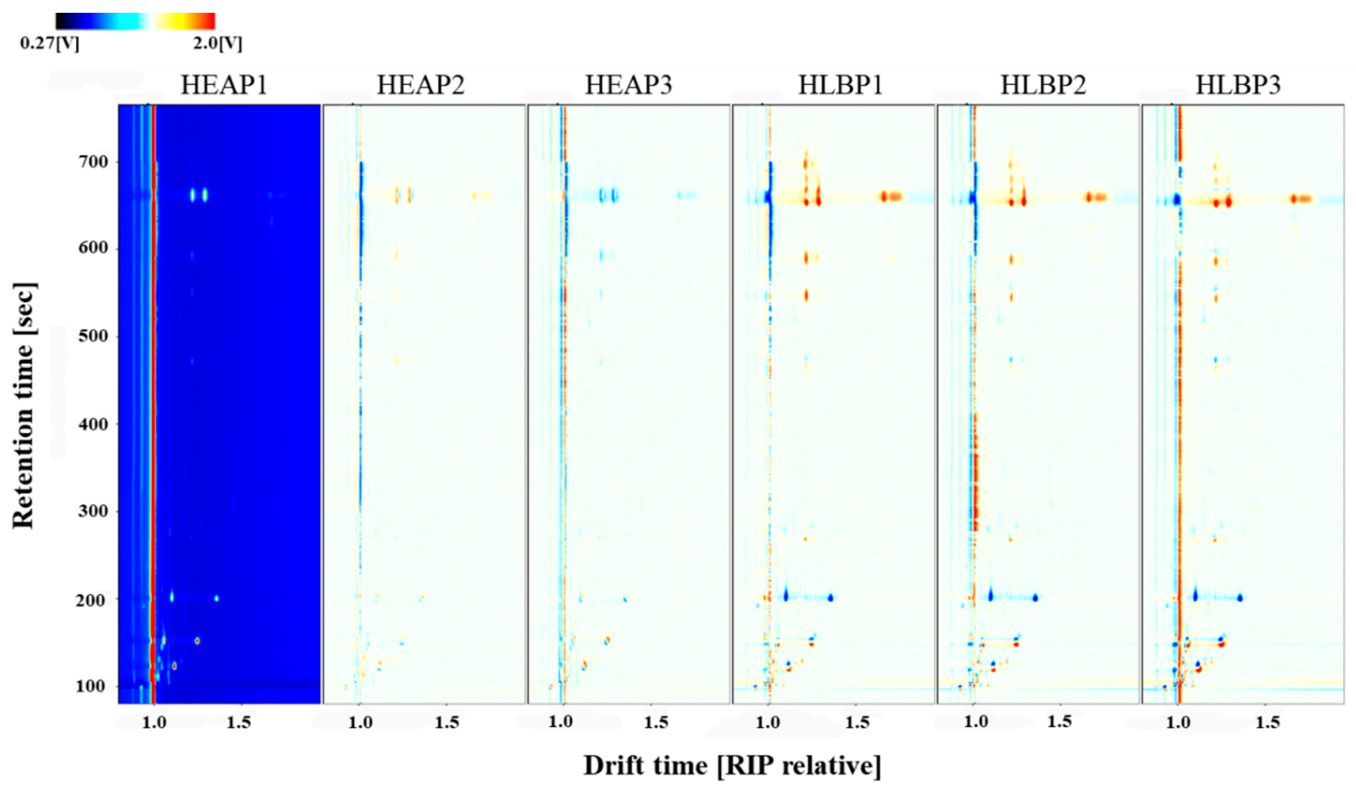

Figure 4. Comparison of ion migration chromatogram of volatile organic compounds from healthy and HLB-infected pomelo leaves (HEAP and HLBP). 


\subsection{Differences in the Characteristic Volatile Fingerprints of $H E A O, H L B O, H E A P$, and HLBP}

Based on the peak signal of the topographic plots, the fingerprints of HEAO and HLBO were generated using the Gallery Plot to accurately evaluate the VOCs, as shown in Figure 5. The full fingerprint of VOCs from the orange leaves HEAO and HLBO was divided into two parts as A and $\mathrm{B}$ for better comparison. The full fingerprint of VOCs from the pomelo leaves HEAP and HLBP is presented in part $\mathrm{C}$.
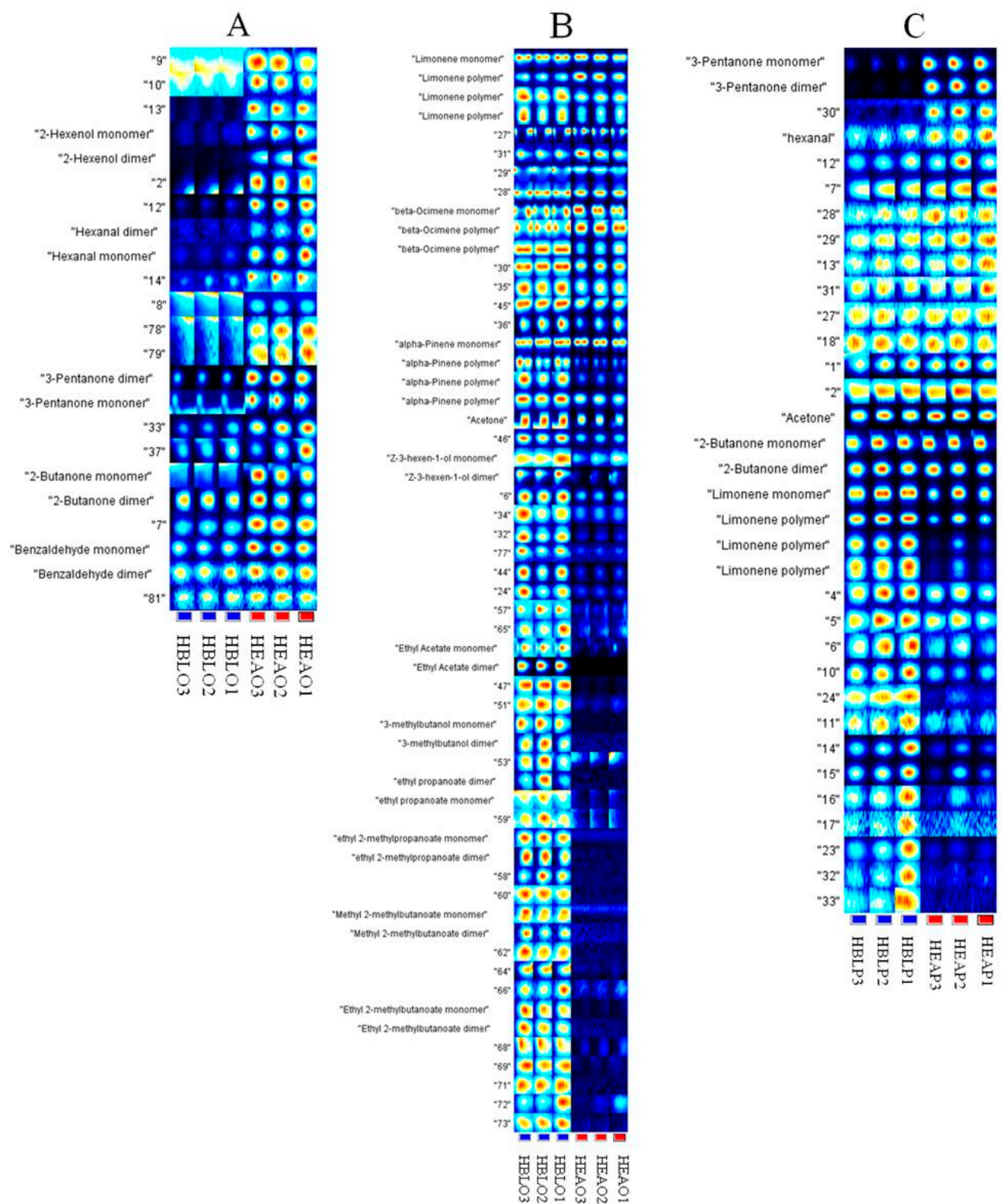

Figure 5. Two parts (A,B) of the fingerprints of HEAO and HLBO samples and the full fingerprints (part $(\mathbf{C})$ ) of HEAP and HLBP generated using the Gallery.

In the fingerprint, each row represents the entire signal peak of one sample, and each column represents the same VOC in different samples. The content of VOCs is distinguished by colors. The higher the content, the brighter the color. VOCs with the same name in the fingerprints are presented as monomers, dimers, or polymers. The drift time of dimers or polymers was increased due to their proton affinity and higher content [27]. The composition and contents of VOCs in HEAO and HLBO can be compared intuitively using fingerprints. Unfortunately, some VOCs were not identified, 
due to the limited data library. A whole VOC profile should be seen using GC-MS data and HS-GC-IMS data together.

As shown in Figure 5A, ten peaks, including hexanal, 3-pentanone, and 2-butanone, were identified. The brightness of the fingerprint in part A was much stronger in the HEAO fingerprint than that of HLBO. The numbers of identified VOCs in HEAO were more than those in HLBO. Some VOCs, such as 2-hexanol and its dimer, appeared in HEAO, while their fingerprint information in HLBO was minimal. In addition, 3-pentanone was present in HLBO; however, the brightness of its fingerprint was much weaker than that in HEAO. As shown in Figure 5B, twenty-six peaks, including terpenes (limonene, $\alpha$-pinene, and $\beta$-ocimene) and other VOCs were identified. Most peaks in Part B showed a much brighter fingerprint in HLBO than that of in HEAO.

The fingerprints of HEAP and HLBP were generated using the Gallery Plot to accurately evaluate the VOCs in pomelo leaves, as shown in Figure 5C. Ten peaks, including hexanal, 3-pentanone, 2-butanone, and limonene, were identified. Most peaks showed a much brighter fingerprint in HLBP than that in HEAP.

\subsection{Identification of Volatile Organic Compounds in HEAO, HLBO, HEAP, and HLBP}

The qualitative analysis of VOCs in HEAO and HLBO is represented in Table 1 and Figure 6. Some VOCs presented multiple signals as monomers, dimers, and polymers, due to their varying concentrations and adducts formation while moving through the IMS drift tube [27]. These VOCs had the same GC retention times, but different drift times. Table 1 lists all the identified VOCs from the GC-IMS library in orange leaf samples, including the compound name, retention index (RI), retention time (Rt), drift time (Dt) (RIP relative), and signal intensity (SI). RI values were calculated using the homologous series of n-2-ketones C4-C9: 2-butanone, 2-pentanone, 2-hexanone, 2-heptanone, 2-octanone, and 2-nonanone, as external standard on the FS-SE-54-CB capillary column. Acetone should be excluded for further analysis because it might come from the cleaning agent. As shown in Figure 6, the two-dimensional topographic plots of VOCs in HEAO and HLBO were obtained at the retention time and the normalized drift time by HS-GC-IMS. Each marked dot represents a type of identified VOC with the same serial number presented in Table 1 . The higher the intensity of the red color, the higher the concentration of VOCs; the blue color has the opposite interpretation. These plots show some visual differences of VOCs by location and relative content between healthy and HLB-infected orange leaves.

Highly significant differences $(p<0.001)$ in signal intensity between HEAO and HLBO were observed for 3-pentanone and its dimer, ethyl 2-methylbutanoate, limonene, $\alpha$-pinene, ethyl acetate and its dimer, ethyl 2-methylpropanoate, benzaldehyde, and methyl 2-methylbutanoate. For each compound identified, the percent difference of average signal intensity between HEAO and HLBO samples was compared (Table 1). The following equation for percent difference was utilized:

$$
\text { Difference }=\left[\left(\mathrm{SI}_{\mathrm{HAEO}}-\mathrm{SI}_{\mathrm{HLBO}}\right) /\left(\mathrm{SI}_{\mathrm{HAEO}}\right)\right] \times 100 \%
$$

The largest percent differences (higher than $300 \%$ ) were for ethyl acetate dimer (5733.5\%), 3-methylbutanol (684.9\%), ethyl 2-methylbutanoate (611.6\%), ethyl 2-methylpropanoate dimer $(402.8 \%)$, ethyl 2-methylbutanoate dimer (380.3\%), and ethyl propanoate dimer (317.6\%). Of the compounds for which there was a highly significant difference, the HEAO signal intensity was higher for 3-pentanone, 3-pentanone dimer, and benzaldehyde. Conversely, the HLBO signal intensity was higher for ethyl 2-methylbutanoate, limonene, $\alpha$-pinene, ethyl acetate and its dimer, ethyl 2-methylpropanoate, and methyl 2-methylbutanoate. VOCs showing a significant difference in signal intensity in both leaves might be possible indicators for detection of HLB. Representative VOCs showing a highly significant difference $(P<0.001)$ or the largest percent difference $(>300 \%)$ of signal intensity in healthy and HLB-infected leaves are shown in Figure 7. 
Table 1. Headspace-gas chromatography ion mobility spectrometry (HS-GC-IMS) data of volatile organic compounds from healthy and HLB-infected orange leaves (HEAO and HLBO).

\begin{tabular}{|c|c|c|c|c|c|c|c|c|}
\hline NO. & Compound & $\mathrm{RI}^{\mathrm{a}}$ & $\operatorname{Rt}^{\mathrm{b}}(\mathrm{s})$ & $\mathrm{Dt}^{\mathrm{c}}$ (RIP ${ }^{\mathrm{d}}$ Relative) & Signal Intensity(HEAO) & Signal Intensity(HLBO) & $P$ Value & Difference \\
\hline 1 & Acetone & 527.0 & 116.749 & 1.1172 & $2181.6 \pm 48.5$ & $4403.7 \pm 96.3$ & $<0.001$ & $-101.9 \%$ \\
\hline 2 & 2-Butanone & 604.3 & 145.839 & 1.0579 & $731.7 \pm 89.3$ & $481.9 \pm 12.0$ & 0.009 & $34.1 \%$ \\
\hline 3 & 2-Butanone dimer & 601.6 & 144.824 & 1.2477 & $455.2 \pm 91.4$ & $459.9 \pm 34.8$ & 0.937 & $-1.0 \%$ \\
\hline 4 & 3-Pentanone & 702.1 & 195.225 & 1.1063 & $1873.4 \pm 100.9$ & $1129.3 \pm 24.6$ & $<0.001$ & $39.7 \%$ \\
\hline 5 & 3-Pentanone dimer & 700.2 & 193.872 & 1.3535 & $4201.6 \pm 131.5$ & $1307.0 \pm 55.1$ & $<0.001$ & $68.9 \%$ \\
\hline 6 & Hexanal & 790.6 & 273.363 & 1.2527 & $259.4 \pm 43.1$ & $79.7 \pm 11.7$ & 0.002 & $69.3 \%$ \\
\hline 7 & Hexanal dimer & 790.7 & 273.385 & 1.5642 & $51.5 \pm 15.4$ & $24.3 \pm 1.9$ & 0.039 & $52.8 \%$ \\
\hline 8 & 2-Hexenol & 850.6 & 336.576 & 1.1795 & $947.0 \pm 99.2$ & $288.1 \pm 70.8$ & 0.001 & $69.6 \%$ \\
\hline 9 & 2-Hexenol dimer & 850.2 & 336.033 & 1.5212 & $158.5 \pm 45.2$ & $24.4 \pm 4.9$ & 0.035 & $84.6 \%$ \\
\hline 10 & Ethyl 2-methylbutanoate & 847.7 & 333.085 & 1.2394 & $32.1 \pm 7.2$ & $228.1 \pm 18.8$ & $<0.001$ & $-611.6 \%$ \\
\hline 11 & Ethyl 2-methylbutanoate dimer & 848.5 & 334.014 & 1.6553 & $25.7 \pm 7.4$ & $123.5 \pm 21.3$ & 0.002 & $-380.3 \%$ \\
\hline 12 & (Z)-3-Hexen-1-ol & 856.1 & 343.25 & 1.2314 & $189.0 \pm 33.3$ & $285.5 \pm 37.3$ & 0.029 & $-51.1 \%$ \\
\hline 13 & (Z)-3-Hexen-1-ol dimer & 856.1 & 343.204 & 1.512 & $531.2 \pm 247.8$ & $1457.6 \pm 308.6$ & 0.015 & $-174.4 \%$ \\
\hline 14 & Limonene & 1028.8 & 648.135 & 1.2181 & $2292.8 \pm 3.8$ & $2861.4 \pm 73.7$ & $<0.001$ & $-24.8 \%$ \\
\hline 15 & Limonene polymer & 1031.0 & 652.017 & 1.2961 & $2319.9 \pm 190.8$ & $1650.0 \pm 65.9$ & 0.005 & $28.9 \%$ \\
\hline 16 & Limonene polymer & 1031.0 & 652.017 & 1.6592 & $1170.7 \pm 41.8$ & $1841.1 \pm 230.1$ & 0.008 & $-57.3 \%$ \\
\hline 17 & Limonene polymer & 1029.9 & 650.076 & 1.7295 & $1296.3 \pm 115.4$ & $1976.2 \pm 276.4$ & 0.017 & $-52.5 \%$ \\
\hline 18 & $\alpha$-Pinene & 929.7 & 458.139 & 1.2185 & $3590.8 \pm 44.2$ & $4499.7 \pm 133.8$ & $<0.001$ & $-25.3 \%$ \\
\hline 19 & $\alpha$-Pinene polymer & 930.0 & 458.63 & 1.2923 & $698.4 \pm 12.3$ & $1144.4 \pm 70.0$ & 0.007 & $-63.9 \%$ \\
\hline 20 & $\alpha$-Pinene polymer & 927.9 & 454.705 & 1.6744 & $1947.1 \pm 150.8$ & $5740.3 \pm 1061.1$ & 0.004 & $-194.8 \%$ \\
\hline 21 & $\alpha$-Pinene polymer & 930.2 & 459.12 & 1.7323 & $269.3 \pm 31.1$ & $808.4 \pm 137.0$ & 0.003 & $-200.2 \%$ \\
\hline 22 & Ethyl acetate & 615.6 & 150.24 & 1.0973 & $292.9 \pm 39.2$ & $1091.6 \pm 26.0$ & $<0.001$ & $-272.7 \%$ \\
\hline 23 & Ethyl acetate dimer & 615.6 & 150.24 & 1.3355 & $105.2 \pm 19.4$ & $6139.0 \pm 49.9$ & $<0.001$ & $-5733.5 \%$ \\
\hline 24 & Ethyl propanoate & 711.9 & 202.517 & 1.1434 & $69.9 \pm 6.4$ & $220.5 \pm 28.1$ & 0.001 & $-215.7 \%$ \\
\hline 25 & Ethyl propanoate dimer & 710.6 & 201.527 & 1.4548 & $14.3 \pm 0.8$ & $59.8 \pm 22.6$ & 0.073 & $-317.6 \%$ \\
\hline 26 & Ethyl 2-methylpropanoate & 754.3 & 238.813 & 1.1905 & $45.9 \pm 9.3$ & $172.1 \pm 4.6$ & $<0.001$ & $-274.8 \%$ \\
\hline 27 & Ethyl 2-methylpropanoate dimer & 752.1 & 236.833 & 1.5619 & $11.6 \pm 0.2$ & $58.4 \pm 5.6$ & 0.005 & $-402.8 \%$ \\
\hline 28 & Benzaldehyde & 953.0 & 503.079 & 1.1434 & $608.3 \pm 14.2$ & $427.2 \pm 21.4$ & $<0.001$ & $29.8 \%$ \\
\hline 29 & Benzaldehyde dimer & 953.0 & 503.193 & 1.47 & $101.6 \pm 2.3$ & $85.2 \pm 10.3$ & 0.104 & $16.2 \%$ \\
\hline 30 & 3-Methylbutanol & 734.6 & 221.157 & 1.2418 & $32.4 \pm 3.4$ & $254.5 \pm 23.0$ & 0.003 & $-684.9 \%$ \\
\hline 31 & 3-Methylbutanol dimer & 734.1 & 220.7 & 1.4927 & $18.5 \pm 2.8$ & $72.1 \pm 11.1$ & 0.001 & $-289.1 \%$ \\
\hline 32 & Methyl 2-methylbutanoate & 770.0 & 253.595 & 1.1895 & $59.6 \pm 3.9$ & $157.8 \pm 13.0$ & $<0.001$ & $-164.7 \%$ \\
\hline 33 & Methyl 2-methylbutanoate dimer & 770.0 & 253.595 & 1.5347 & $21.5 \pm 1.0$ & $49.5 \pm 7.8$ & 0.004 & $-129.8 \%$ \\
\hline 34 & $\beta$-Ocimene & 1052.9 & 690.729 & 1.2133 & $3753.4 \pm 75.8$ & $3654.0 \pm 51.4$ & 0.133 & $2.6 \%$ \\
\hline 35 & $\beta$-Ocimene polymer & 1051.9 & 688.902 & 1.2566 & $1533.9 \pm 146.8$ & $1386.7 \pm 103.9$ & 0.229 & $9.6 \%$ \\
\hline 36 & $\beta$-Ocimene polymer & 1052.4 & 689.815 & 1.6976 & $3809.0 \pm 1056.0$ & $7601.4 \pm 113.5$ & 0.003 & $-99.6 \%$ \\
\hline
\end{tabular}

$\mathrm{RI}^{\mathrm{a}}$ : Retention index; $\mathrm{Rt}^{\mathrm{b}}$ : Retention time; $\mathrm{Dt}^{\mathrm{c}}$ : Drift time (RIP relative); RIP ${ }^{\mathrm{d}}$ : Reactant ion peak. Values of signal intensity are given as mean $\pm \mathrm{SD}(\mathrm{n}=3) . P<0.05$ : Significant, $P<0.01$ : Very significant, and $P<0.001$ : Highly significant. 

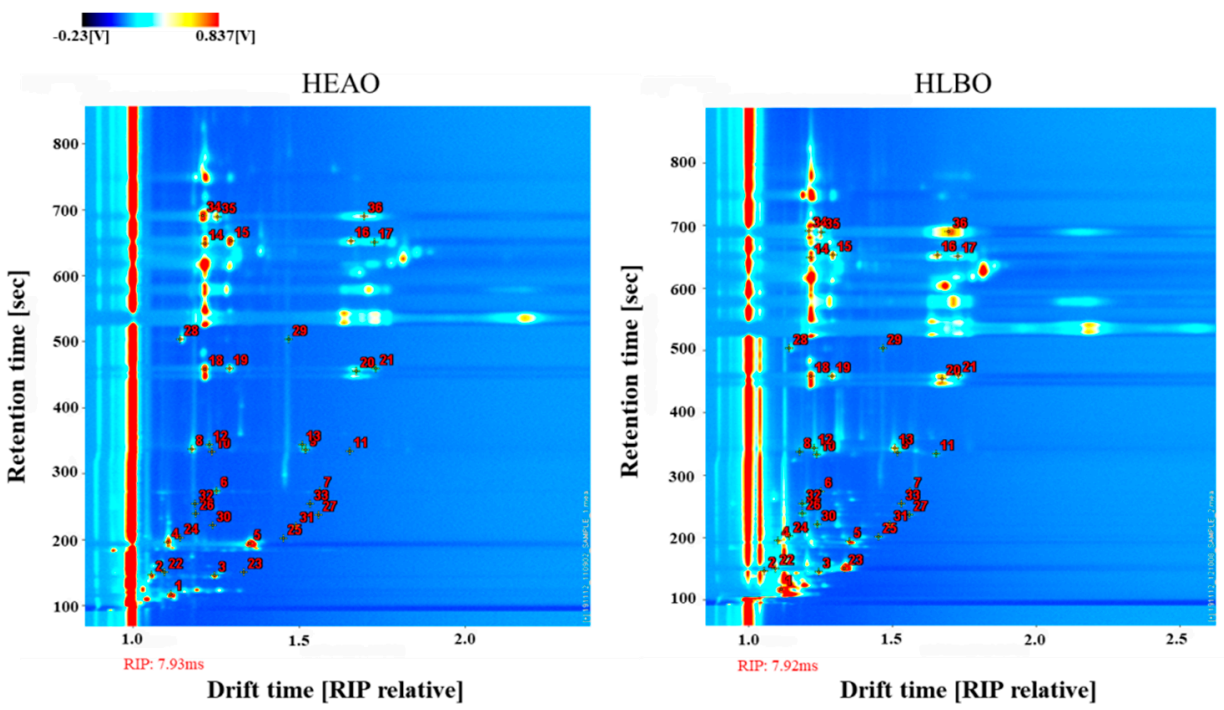

Figure 6. Two-dimensional topographic plots of VOCs in HEAO and HLBO obtained at the retention time and the normalized drift time by HS-GC-IMS. Each marked dot represents a type of identified VOC with the same serial number presented in Table 1.

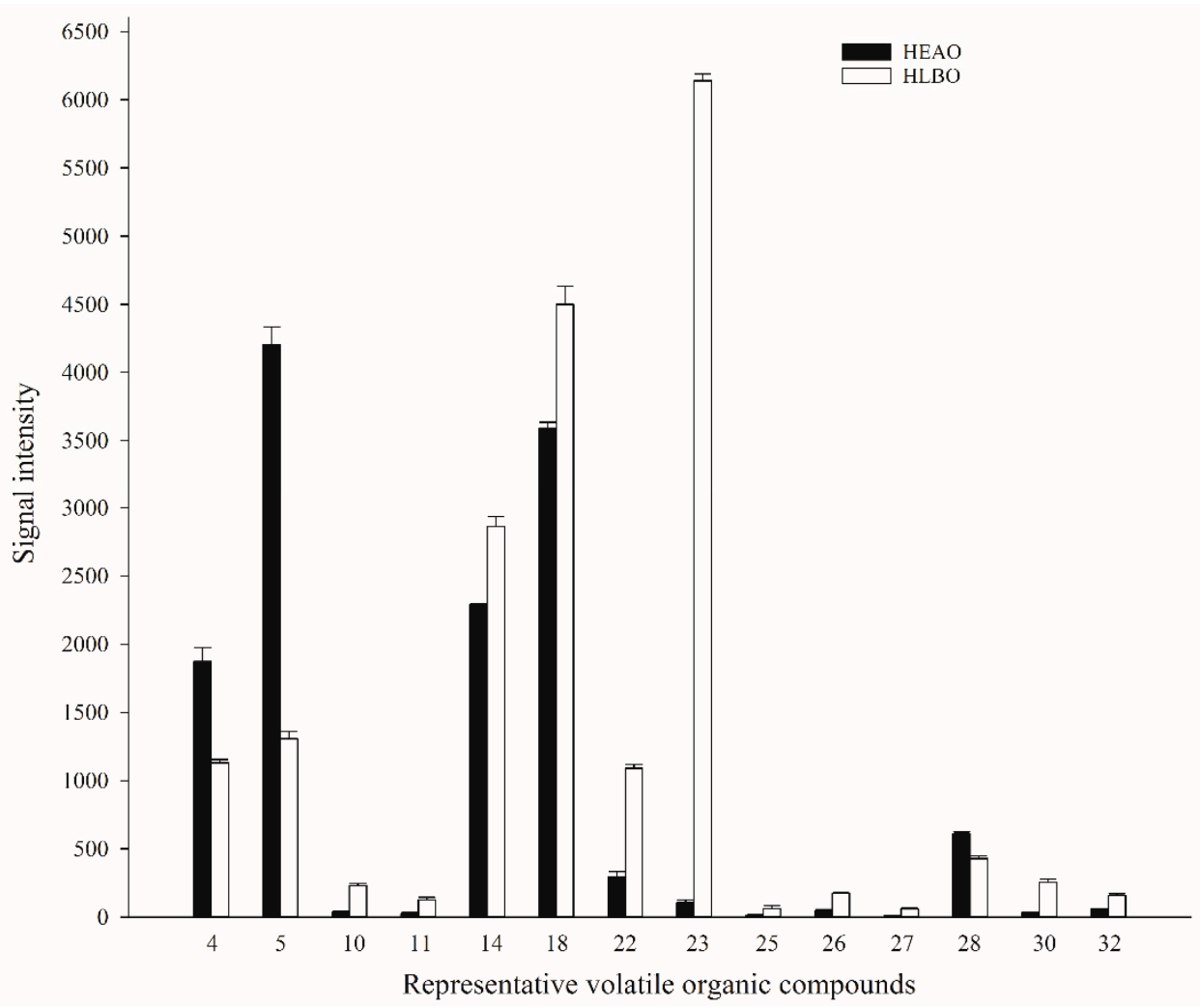

Figure 7. Representative VOCs showing highly significant difference $(p<0.001)$ or the largest percent difference $(>300 \%)$ of signal intensity in healthy and HLB-infected leaves. The numbers on the Y-axis denote the same compound numbers in Table 1.

The qualitative analysis of VOCs in HEAP and HLBP is represented in Table 2 and Figure 8. Each marked dot in the two-dimensional topographic plot in Figure 8 represents a type of identified VOC with the same serial number presented in Table 2. VOC content was determined by the brightness degree of color. These plots showed some visual differences in VOCs by location and relative content 
between healthy and HLB-infected pomelo leaves. The number of identified characteristic peaks (nine characteristic peaks excluding acetone) from the GC-IMS library in pomelo leaf samples was less than that of orange leaf (35 characteristic peaks excluding acetone). The signal intensity of 3-pentanone, 3-pentanone dimer, and limonene polymer has shown a highly significant difference $(p<0.001)$ between healthy and HLB-infected Shatian pomelo leaves. However, the signal intensity of 2-butanone, 2-butanone dimer, and hexanal did not show a significant difference between HEAP and HLBP. For each compound identified, the percent difference of average signal intensity between HEAP and HLBP samples was compared. The following equation was used for percent difference:

$$
\text { Difference }=\left[\left(\mathrm{SI}_{\mathrm{HAEP}}-\mathrm{SI}_{\mathrm{HLBP}}\right) /\left(\mathrm{SI}_{\mathrm{HAEP}}\right)\right] \times 100 \%
$$

Table 2. HS-GC-IMS data of volatile organic compounds from healthy and HLB-infected pomelo leaves (HEAP and HLBP).

\begin{tabular}{|c|c|c|c|c|c|c|c|c|}
\hline No. & Compound & $R I^{a}$ & $\mathrm{Rt}^{\mathrm{b}}(\mathrm{s})$ & $\mathrm{Dt}^{\mathrm{c}}$ (RIP ${ }^{\mathrm{d}}$ Relative) & Signal Intensity(HEAP) & Signal Intensity(HLBP) & $P$ Value & Difference \\
\hline 1 & 2-Butanone & 617.4 & 150.906 & 1.0595 & $1220.5 \pm 47.5$ & $1307.7 \pm 106.4$ & 0.265 & $-7.1 \%$ \\
\hline 2 & 2-Butanone dimer & 616.7 & 150.640 & 1.2515 & $1404.5 \pm 131.9$ & $1589.0 \pm 108.2$ & 0.135 & $-13.1 \%$ \\
\hline 3 & 3-Pentanone & 712.0 & 202.578 & 1.1028 & $1022.3 \pm 35.8$ & $259.0 \pm 29.2$ & $<0.001$ & $74.7 \%$ \\
\hline 4 & 3-Pentanone dimer & 710.2 & 201.249 & 1.3599 & $648.2 \pm 51.1$ & $51.6 \pm 8.3$ & $<0.001$ & $92.0 \%$ \\
\hline 5 & Acetone & 548.7 & 124.904 & 1.1203 & $1575.1 \pm 83.2$ & $1593.1 \pm 45.3$ & 0.759 & $-1.1 \%$ \\
\hline 6 & hexanal & 800.3 & 282.722 & 1.2521 & $38.8 \pm 4.0$ & $32.0 \pm 3.7$ & 0.093 & $17.5 \%$ \\
\hline 7 & Limonene & 1036.5 & 661.717 & 1.2227 & $1584.6 \pm 401.6$ & $2620.9 \pm 103.3$ & 0.012 & $-65.4 \%$ \\
\hline 8 & Limonene polymer & 1037.0 & 662.589 & 1.2929 & $1997.1 \pm 588.6$ & $3978.6 \pm 120.2$ & 0.005 & $-99.2 \%$ \\
\hline 9 & Limonene polymer & 1036.5 & 661.717 & 1.6643 & $242.3 \pm 116.4$ & $929.6 \pm 60.4$ & 0.001 & $-283.7 \%$ \\
\hline 10 & Limonene polymer & 1036.0 & 660.845 & 1.7345 & $291.5 \pm 103.3$ & $990.3 \pm 50.2$ & $<0.001$ & $-239.7 \%$ \\
\hline
\end{tabular}
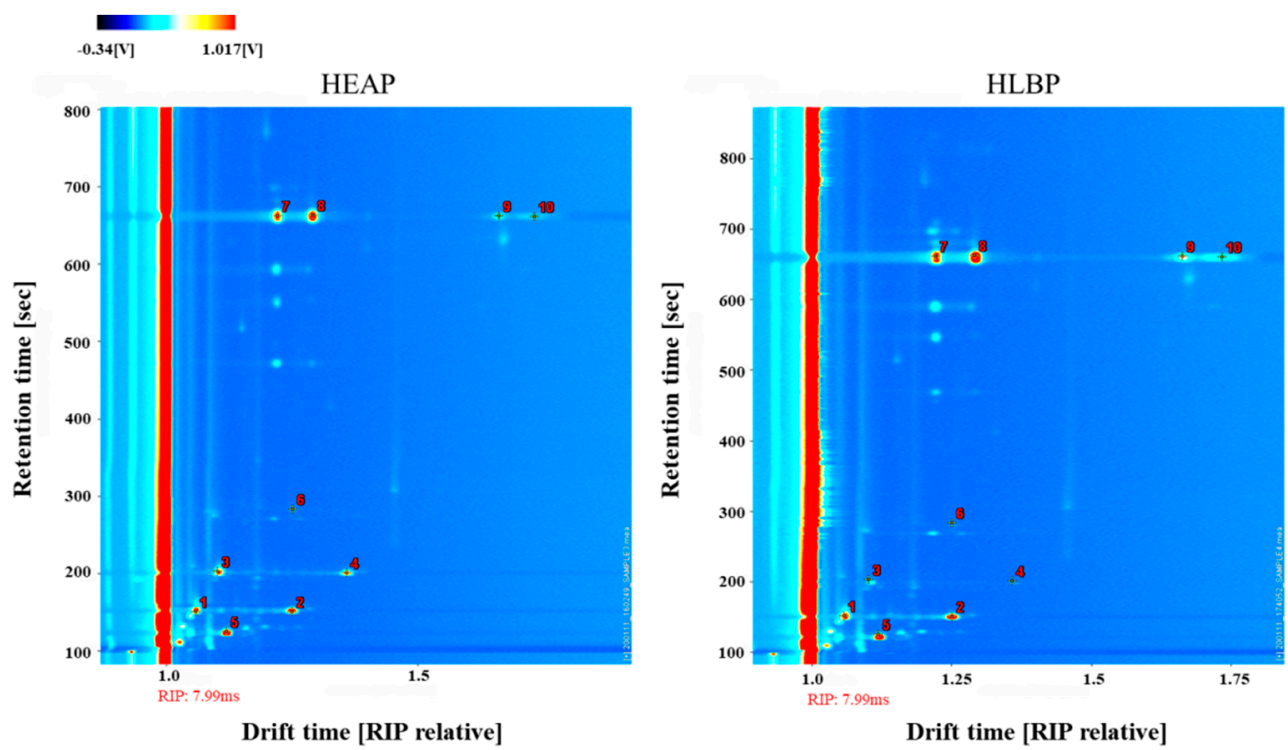

Figure 8. Two-dimensional topographic plots of VOCs in HEAP and HLBP obtained at the retention time and the normalized drift time by HS-GC-IMS. Each marked dot represents a type of identified VOC with the same serial numbers presented in Table 2.

The largest percent differences (higher than 200\%) were for limonene polymer (283.7\% and 239.7\%). These differences could be visually compared in Figure 8 where compounds 9 and 10, which represent limonene polymer in the plot of HLBP, had a brighter color than compounds 9 and 10 in the plot of HEAP. The differences between healthy and HLB-infected Shatian pomelo leaves might provide information for possible indicators for detection of HLB. 


\subsection{Similarity Analysis of Fingerprint Based on PCA}

Principal component analysis (PCA) is a multivariate statistical analysis technique. By determining a few principal component factors to represent many complex variables in the samples, the regularity and difference among samples could be evaluated according to the contribution of principal component factors [30]. PCA was established using signal intensity to highlight the differences of VOCs in HEAO and HLBO samples, as shown in Figure 9. The distribution map for the first two principal components determined by PCA is displayed, which describes $86 \%$ and $8 \%$ of the accumulative variance contribution rate, and a visualization map was obtained. The PCA results clearly show that HEAO (sample 1) and HLBO (sample 2) in a completely independent space would be well-distinguished in the visualization map. HEAO could be well-distinguished according to the positive score values of PC1, while HLBO could be well-defined according to the negative scores of PC1, and the difference in HEAO and HLBO could be distinguished by combining with the score values of PC2.

PCA of the VOCs in HEAP and HLBP samples is shown in Figure 10. The distribution map for the first two principal components is displayed, which describes $69 \%$ and $13 \%$ of the accumulative variance contribution rate. The PCA results clearly show that HEAP (sample 3) and HLBP (sample 4) in a completely independent space would be well-distinguished in the visualization map. HEAP could be well-distinguished according to the positive score values of PC1, while HLBP could be well-defined according to the negative scores of PC1, and the difference in HEAP and HLBP could be distinguished by combining with the score values of PC2.

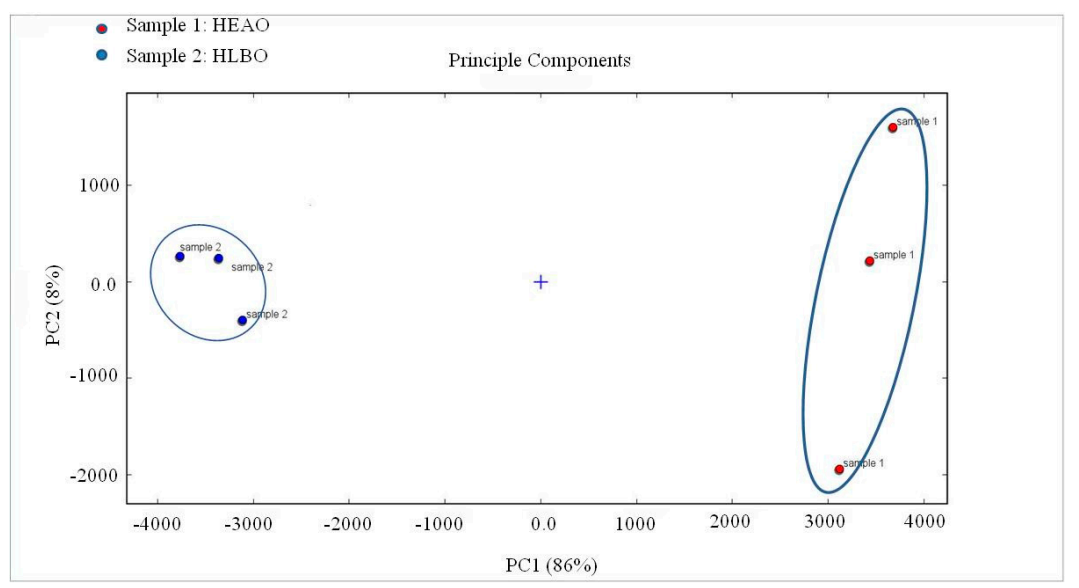

Figure 9. Principal component analysis (PCA) of HEAO (sample 1) and HLBO (sample 2).

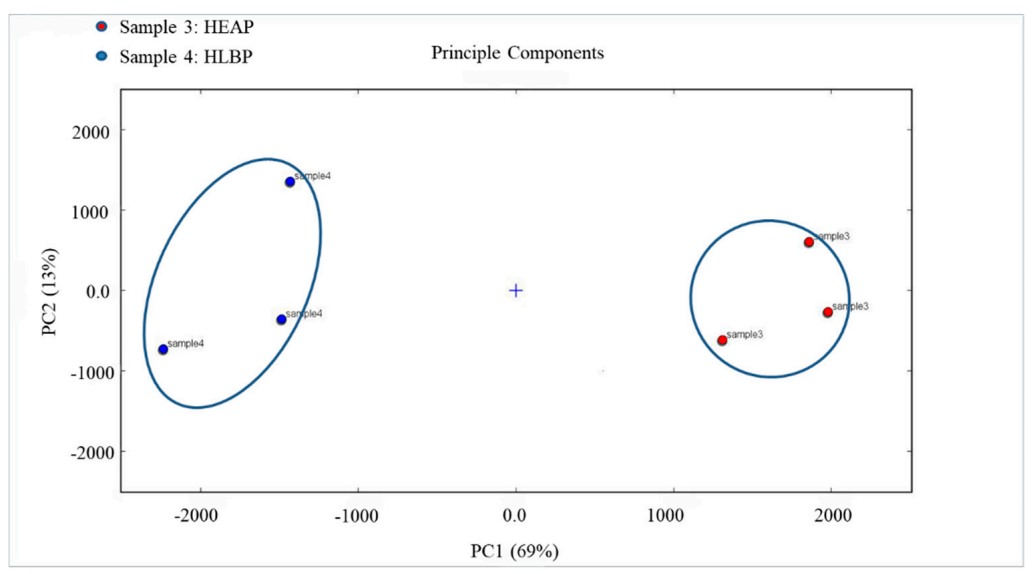

Figure 10. Principal component analysis (PCA) of HEAP (sample 3) and HLBP (sample 4). 


\section{Materials and Methods}

\subsection{Materials}

Gannan Newhall navel orange and Shatian pomelo (Citrus maxima (Burm.) Merr. cv. Shatian Yu) young leaves were used as the experiment material and collected in November 2019 and January 2020, respectively, from the orchard of Gannan Normal University, Ganzhou City in Jiangxi Province, China. The HLB-infected leaves were tested by the polymerase chain reaction (PCR) method.

\subsection{GC-IMS Analyses}

\subsubsection{Apparatuses}

Analyses of samples were completed on a combined device of an Agilent 490 gas chromatograph (Agilent Technologies, Palo Alto, CA, USA) using a FS-SE-54-CB capillary column $(15 \mathrm{~m} \times 0.53 \mathrm{~mm})$, and an IMS instrument Flavor Spec ${ }^{\circledR}$ (Gesellschaft für Analytische Sensorsysteme mbH, Dortmund, Germany), equipped with an autosampler unit (CTC Analytics AG, Zwingen, Switzerland), was used in this study.

\subsubsection{HS-GC-IMS Analysis Methods}

The analysis method was performed as described by Yang et al. [27]. Fresh leaf ( $1 \mathrm{~g}$, without any pretreatment) was cut into small pieces and transferred to a $20 \mathrm{~mL}$ headspace vial and then incubated at $40{ }^{\circ} \mathrm{C}$ for $20 \mathrm{~min}$. Then, a $200 \mu \mathrm{L}$ headspace was injected into the heated injector using a syringe at $85^{\circ} \mathrm{C}$. Nitrogen $(99.99 \%$ purity) was used as the carrier gas. The sample was driven into an FS-SE-54-CB capillary column $(15 \mathrm{~m} \times 0.53 \mathrm{~mm})$ by nitrogen at the following programmed flow: $2 \mathrm{~mL} / \mathrm{min}$ for $2 \mathrm{~min}$, $10 \mathrm{~mL} / \mathrm{min}$ for $10 \mathrm{~min}, 100 \mathrm{~mL} / \mathrm{min}$ for $10 \mathrm{~min}$, and $150 \mathrm{~mL} / \mathrm{min}$ for $30 \mathrm{~min}$. The analytes were separated at $40{ }^{\circ} \mathrm{C}$ in the column and then ionized in the IMS ionization chamber at $45^{\circ} \mathrm{C}$. Drift gas flow was set at a constant flow of $150 \mathrm{~mL} / \mathrm{min}$. All analyses were performed in triplicate. VOCs were identified by comparing retention index (RI) and the drift time (the time taken for ions to reach the collector through the drift tube, in milliseconds) standard in the GC-IMS library (Gesellschaft für Analytische Sensorsysteme $\mathrm{mbH}$, Dortmund, Germany).

\subsection{Statistical Analysis}

The analytical software included a Laboratory Analytical Viewer (LAV, Dortmund, Germany), three plug-ins (G.A.S. Dortmund, Germany), and a GC-IMS library search. IMS data were acquired and processed using LAV processing software and used to generate the analytical spectrum, where each point represented a VOC. The spectrogram differences were compared using the Reporter plug-in. The differences of fingerprint in different samples were compared via the Gallery Plot plug-in. Qualitative analysis of VOCs was achieved based on the National Institute of Standards and Technology (NIST) and IMS databases from the software's built-in GC-IMS library. Statistical analyses of the differences between mean values obtained for experimental groups were calculated using IBM SPSS Statistics 23.0. (IBM Corp. Released 2015. IBM SPSS Statistics for Windows, Version 23.0. Armonk, NY, USA). $p$ values were calculated using a $t$-test between healthy and HLB-infected leaves for each compound. $p$ values $<0.05$ were regarded as significant, $p$ values $<0.01$ as very significant, and $p$ values $<0.001$ as highly significant.

\section{Conclusions}

As plant leaves are a major source of VOCs emitted in the atmosphere and plant foliar VOCs are very important in mediating plant-plant and plant-insect communication, many methods and analytical techniques have been developed for plant foliar VOC research [31]. Comparison of VOCs in navel orange and pomelo healthy and HLB-infected young leaves would be helpful to understand the role of VOCs played in the host plant of ACP, which may be beneficial in designing ACP control 
strategies, as well as HLB detection. In this study, VOCs of HEAO, HLBO, HEAP, and HLBP were identified and analyzed from topographic plots by the HS-GC-IMS technique. The signal intensity of some VOCs in HLBO and HLBP showed a highly significant difference compared to those in HEAO and HEAP, respectively. HLB-infected leaves emitted more VOCs than healthy leaves. These findings were in accordance with the phenomenon where plants tend to increase VOC emissions after herbivore attack [32-34]. The PCA results clearly showed that HEAO and HLBO, as well as HEAP and HLBP, were in a relatively independent space and were well-distinguished. A novel method was developed to evaluate the characteristic VOCs of orange leaf samples by establishing the fingerprint with HS-GC-IMS and PCA. As well as we know, using HS-GC-IMS to analyze healthy and HLB-infected orange and pomelo young leaves has not been reported by other research groups. Taken together, information of VOCs identified by the HS-GC-IMS fingerprint and PCA could be a useful tool for the identification and classification of orange and pomelo leaf samples. Our study may help develop new strategies for the detection of HLB or find new attractants or repellents of ACP for prevention of HLB. It may also help explore plant-insect and plant-pathogen communication under biotic stresses. Unfortunately, many VOCs were not identified, due to the limited data library, especially for pomelo leaf samples. The development of a data library of HS-GC-IMS and more synergistic methods and approaches are expected for plant foliar VOC research in the future.

Author Contributions: J.C. and B.Z. contributed to the conception and design of the study and data analysis; S.C., J.S., X.Y., W.D. and J.C. collected the plant material and performed investigation and data analysis; J.C., S.C. and J.S. wrote the manuscript. All authors have read and agreed to the published version of the manuscript.

Funding: This work was supported by the National Natural Science Foundation of China (31960549), Foundation of Jiangxi Province Educational Committee (KJLD14079), and Natural Science Foundation of Jiangxi Province (20181BBF60030).

Acknowledgments: We thank Chunyan Zhang and Benyou Wang (Shandong Hanon Scientific Instruments Co., Ltd.) for the technique help for HS-GC-IMS.

Conflicts of Interest: The authors declare no conflict of interest.

\section{References}

1. Wang, N.; Pierson, E.A.; Setubal, J.C.; Xu, J.; Levy, J.G.; Zhang, Y.; Li, J.; Rangel, L.T.; Martins, J.J. The Candidatus liberibacter-host interface: Insights into pathogenesis mechanisms and disease control. Annu. Rev. Phytopathol. 2017, 55, 451-482. [CrossRef]

2. $\quad$ Singerman, A. The Real Cost of HLB in Florida. Citrus Ind. 2019, 2019, 10-13.

3. Rao, M.J.; Ding, F.; Wang, N.; Deng, X.; Xu, Q. Metabolic Mechanisms of Host Species against Citrus Huanglongbing (Greening Disease). Crit. Rev. Plant Sci. 2019, 37, 496-511. [CrossRef]

4. Xu, B.M.; Baker, G.L.; Sarnoski, P.J.; Goodrich-Schneider, R.M. A Comparison of the Volatile Components of Cold Pressed Hamlin and Valencia (Citrus sinensis (L.) Osbeck) Orange Oils Affected by Huanglongbing. J. Food Qual. 2017, 2017, 6793986. [CrossRef]

5. Yan, H.; Zeng, J.; Zhong, G. The push-pull strategy for citrus psyllid control. Pest Manag. Sci. 2015, 71, 893-896. [CrossRef] [PubMed]

6. Patt, J.; Sétamou, M. Responses of the Asian citrus psyllid to volatiles emitted by the flushing shoots of its rutaceous host plants. Environ. Entomol. 2010, 39, 618-624. [CrossRef] [PubMed]

7. Mann, R.S.; Ali, J.G.; Hermann, S.L.; Tiwari, S.; Pelz-Stelinski, K.S.; Hans, T.; Alborn, H.T.; Stelinski, L.L. Induced release of a plant-defense volatile "deceptively" attracts insect vectors to plants infected with a bacterial pathogen. PLoS Pathog. 2012, 8, e1002610. [CrossRef] [PubMed]

8. Wenninger, E.J.; Stelinski, L.L.; Hall, D.G. Role of olfactory cues, visual cues, and mating status in orientation of Diaphorina citri Kuwayama (Hemiptera: Psyllidae) to four different host-plants. Environ. Entomol. 2009, 38, 225-234. [CrossRef]

9. Robbins, P.S.; Alessandro, R.T.; Stelinski, L.L.; Lapointe, S.L. Volatile profiles of young leaves of Rutaceae spp. Varying in susceptibility to the Asian citrus psyllid (Hemiptera: Psyllidae). Fla. Entomol. 2012, 95, 774-776. [CrossRef] 
10. Alquézar, B.; Volpe, H.X.L.; Magnani, R.F.; Miranda, M.P.D.; Santos, M.A.; Wulff, N.A.; Bento, J.M.S.; Parra, J.R.P.; Bouwmeester, H.; Peňa, L. $\beta$-Caryophyllene emitted from a transgenic Arabidopsis or chemical dispenser repels Diaphorina citri, vector of Candidatus Liberibacters. Sci. Rep. 2017, 7, 5639. [CrossRef]

11. Andrade, M.D.S.; Ribeiro, L.d.P.; Borgoni, P.C.; da Silva, M.F.; Forim, M.R.; Fernandes, J.B.; Vieira, J.D.; Vendramin, J.D.; Machado, M.A. Essential oil variation from twenty two genotypes of Citrus in Brazil-chemometric approach and repellency against Diaphorina citri Kuwayama. Molecules 2016, 21, 814. [CrossRef]

12. Chen, H.; Palmer, I.A.; Chen, J.; Chang, M.; Thompson, S.L.; Liu, L.; Fu, Z. Specific and Accurate Detection of the Citrus Greening Pathogen Candidatus liberibacter spp. Using Conventional PCR on Citrus Leaf Tissue Samples. J. Vis. Exp. 2018, 136, e57240. [CrossRef] [PubMed]

13. Iftikhar, Y.; Rauf, S.; Shahzad, U.; Zahid, M.A. Huanglongbing: Pathogen detection system for integrated disease management-A review. J. Saudi Soc. Agric. Sci. 2016, 15, 1-11. [CrossRef]

14. Mendoza-Peña, E.; Cibrián-Tovar, J.; Velázquez-González, J.; Tafoya-Rangel, F.; Azuara-Domínguez, F. Volatile compounds of Persian and Mexican lime associated with HLB (Huanglongbing) symptoms. Rev. Colomb. Entomol. 2018, 44, 19-24. [CrossRef]

15. Aksenov, A.A.; Martini, X.; Zhao, W.; Stelinski, L.L.; Davis, C.E. Synthetic blends of volatile, phytopathogen-induced odorants can be used to manipulate vector behavior. Front. Ecol. Evol. 2014, 2, 1-9. [CrossRef]

16. Wang, H.; Ramnani, P.; Pham, T.; Villarreal, C.C.; Yu, X.; Liu, J.; Mulchandani, A. Gas Biosensor Arrays Based on Single-Stranded DNA-Functionalized Single-Walled Carbon Nanotubes for the Detection of Volatile Organic Compound Biomarkers Released by Huanglongbing Disease-Infected Citrus Trees. Sensors 2019, 19, 4795. [CrossRef]

17. Aksenov, A.A.; Pasamontes, A.; Peirano, D.J.; Zhao, W.; Dandekar, A.M.; Fiehn, O.; Ehsani, R.; Davis, C.E. Detection of Huanglongbing disease using differential mobility spectrometry. Anal. Chem. 2014, 86, 2481-2488. [CrossRef]

18. U.S. Department of Agriculture (USDA). Citrus: World Markets and Trade. Available online: http: //apps.fas.usda.gov/psdonline/circulars/citrus.pdf (accessed on 27 July 2020).

19. Zhang, L.; Geng, Y.; Zhu, H.; Mu, Y.; Yu, J.; Li, J.; Wang, X. Preparative separation of six coumarins from the pummelo (Citrus maxima (Burm.) Merr. Cv. Shatian Yu) peel by high-speed countercurrent chromatography. J. Liq. Chromatogr. Relat. Technol. 2017, 40, 991-996. [CrossRef]

20. Riu-Aumatell, M.; Vargas, L.; Vichi, S.; Guadayol, J.M.; López-Tamames, E.; Buxaderas, S. Characterisation of volatile composition of white salsify (Tragopogon porrifolius L.) by headspace solid-phase microextraction (HS-SPME) and simultaneous distillation-extraction (SDE) coupled to GC-MS. Food Chem. 2011, 129, 557-564. [CrossRef]

21. Peng, F.; Sheng, L.; Liu, B.; Tong, H.; Liu, S. Comparison of different extraction methods: Steam distillation, simultaneous distillation and extraction and headspace co-distillation, used for the analysis of the volatile components in aged flue-cured tobacco leaves. J. Chromatogr. A 2004, 1040, 1-17. [CrossRef]

22. Pourmortazavi, S.M.; Hajimirsadeghi, S.S. Supercritical fluid extraction in plant essential and volatile oil analysis. J. Chromatogr. A 2007, 1163, 2-24. [CrossRef]

23. Wang, Z.; Gao, C.; Liu, J.; Zhou, W.; Zeng, X. Host plant odours and their recognition by the odourant-binding proteins of Diaphorina citri Kuwayama (Hemiptera: Psyllidae). Pest Manag. Sci. 2020, 76, 2453-2464. [CrossRef]

24. Sharma, R.; Zhou, M.; Hunter, M.D.; Fan, X. Rapid In Situ Analysis of Plant Emission for Disease Diagnosis Using a Portable Gas Chromatography Device. J. Agric. Food Chem. 2019, 67, 7530-7537. [CrossRef]

25. Hernández-Mesa, M.; Ropartz, D.; García-Campaña, A.M.; Rogniaux, M.; Dervilly-Pinel, G.; Bizec, B.L. Ion Mobility Spectrometry in Food Analysis: Principles, Current Applications and Future Trends. Molecules 2019, 24, 2706. [CrossRef]

26. Kafle, G.K.; Khot, L.R.; Sankaran, S.; Bahlol, H.Y.; Tufariello, J.A.; Hill, H.H. State of ion mobility spectrometry and applications in agriculture: A review. Eng. Agric. Environ. Food 2016, 9, 346-357. [CrossRef]

27. Yang, L.; Liu, J.; Wang, X.; Wang, X.; Ren, F.; Zhang, Q.; Shan, Y.; Ding, S. Characterization of Volatile Component Changes in Jujube Fruits during Cold Storage by Using Headspace-Gas Chromatography-Ion Mobility Spectrometry. Molecules 2019, 24, 3904. [CrossRef] 
28. Hu, X.; Wang, R.; Guo, J.; Ge, K.; Li, G.; Fu, F.; Ding, S.; Shan, Y. Changes in the Volatile Components of Candied Kumquats in Different Processing Methodologies with Headspace-Gas Chromatography-Ion Mobility Spectrometry. Molecules 2019, 24, 3053. [CrossRef]

29. Rodríguez-Maecker, R.; Vyhmeister, E.; Meisen, S.; Martinez, A.R.; Kuklya, A.; Telgheder, U. Identification of terpenes and essential oils by means of static headspace gas chromatography-ion mobility spectrometry. Anal. Bioanal. Chem. 2017, 409, 6595-6603. [CrossRef]

30. Sebzalli, Y.; Wang, X. Knowledge discovery from process operational data using PCA and fuzzy clustering. Eng. Appl. Artif. Intell. 2001, 14, 607-616. [CrossRef]

31. Materic, D.; Bruhn, D.; Turner, C.; Morgan, G.; Mason, N.; Gauci, V. Methods in plant foliar volatile organic compounds research. Appl. Plant Sci. 2015, 3, 1500044. [CrossRef]

32. Kessler, A.; Baldwin, I.T. Defensive function of herbivore induced plant volatile emissions in nature. Science 2001, 291, 2141-2144. [CrossRef] [PubMed]

33. Heil, M.; Silva Bueno, J.C. Within-plant signaling by volatiles leads to induction and priming of an indirect plant defense in nature. Proc. Natl. Acad. Sci. USA 2007, 104, 5467-5472. [CrossRef] [PubMed]

34. Kessler, A.; Kalske, A. Plant secondary metabolite diversity and species interactions. Annu. Rev. Ecol. Evol. Syst. 2018, 49, 115-138. [CrossRef]

Sample Availability: Samples of Gannan Newhall navel orange leaves (HEAO and HLBO) and Shatian pomelo leaves (HEAP and HLBP) are available from the authors.

(C) 2020 by the authors. Licensee MDPI, Basel, Switzerland. This article is an open access article distributed under the terms and conditions of the Creative Commons Attribution (CC BY) license (http://creativecommons.org/licenses/by/4.0/). 